\title{
Unilateral breast carcinoma with three different histological types-case report
}

\begin{abstract}
Breast cancer is the commonest malignancy in the world Unilateral serous or serosanguinous nipple discharge is always a cause for concern. $10 \%$ of the cases are due to malignant causes. However, we report a rare case of a 52 year old woman with serosanguinous nipple discharge who was later diagnosed with unilateral breast carcinoma with three different histological types which were not detected by mammogram and a MRI Breast was needed to diagnose it. It is a rarity and not reported till date.
\end{abstract}

Keywords: histopathology, MRI breast, malignancy, serosanguinous
Volume 12 Issue 2 - 202 I

\author{
Aditi Shivcharan Agrawal,' Rajshri Kelkar, ${ }^{2}$ \\ Tuhina Mishra ${ }^{3}$ \\ 'Consultant Surgery, Wockhardt Hospital, India \\ ${ }^{2}$ Honorary Consultant Surgery, Grant Government Medical \\ College and Sir J.J Group of Hospitals, India \\ Intern, Grant Government Medical College and Sir J.J Group of \\ Hospitals, India
}

Correspondence: Dr. Aditi Shivcharan Agrawal, Consultant Surgery, Wockhardt hospital, Mira road, Mumbai, India, Email draditiagrawal@gmail.com

Received: April 14, 2021 | Published: April 23, 2021

\section{Introduction}

Breast cancer is the commonest malignancy in the world and accounts for unfavourable outcomes that makes it the second leading cause of cancer death among women. ${ }^{1}$ Unilateral serous or serosanguinous nipple discharge is always a cause for concern. $90 \%$ of the cases it is due to benign causes. However, we report a rare case of a woman with serosanguinous nipple discharge who was later diagnosed with unilateral breast carcinoma with three different histological types which were not detected by mammogram. Inspite of extensive online search, we could not find a similar case and we report one of our kind case.

\section{Case report}

52 year old postmenopausal woman presented with serous nipple discharge from her left breast since one month. There were no other complaints of pain, ulceration or itching. Physically no abnormality except for serosanguinous discharge was detected. X-ray mammogram (Figure 1) was suggestive of dense fibro glandular parenchyma of both breasts with prominent hypoechoic glandular tissue on left breast. Cytological examination of nipple discharge did not reveal any malignant cells. Patient responded to the antibiotics prescribed to her. However, the patient came back after 20 days with episode of spontaneous serosanguinous nipple discharge from her left breast which stained her undergarment. So, with high index of suspicion, MRI (Figure 2) of left breast with breast coils was advised. It revealed micro calcification extending over approx. $9 \mathrm{~cm}$ length of the breast parenchyma. Vacuum assisted biopsy was performed and histopathological examination of the microcalcification revealed mucinous carcinoma and intraductal papillary carcinoma and invasive ductal carcinoma at the same time (Figure 3). Furthermore, immunohistochemical examination revealed that it had positive expression for estrogen receptor (ER) and progesterone receptor (PR) but her HER-2 neu was negative. Patient was counselled and owing to the spread of micro calcification she was suggested to undergo Modified radical mastectomy. Final specimen revealed $22 \times 22 \times 6 \mathrm{~cm}$ mass and the presence of mucinous carcinoma, intraductal papillary carcinoma and invasive duct carcinoma. Apical, axillary and intraductal lymph node were negative for metastasis.

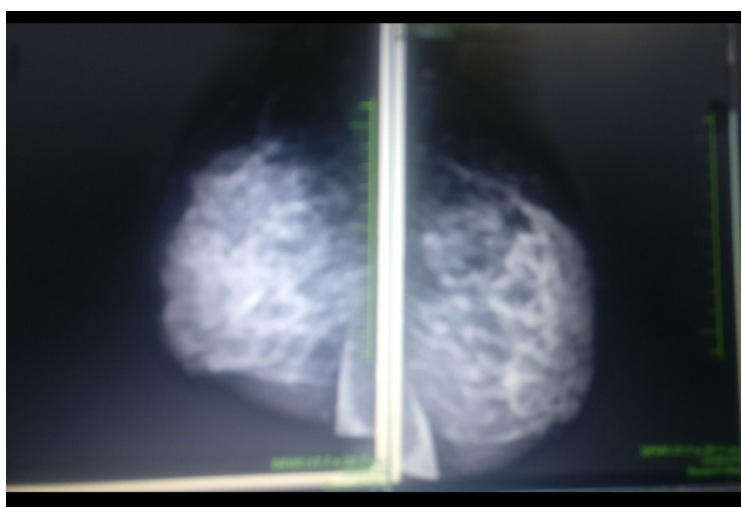

Figure I Mammogram showing dense fibro glandular parenchyma of both the breasts.

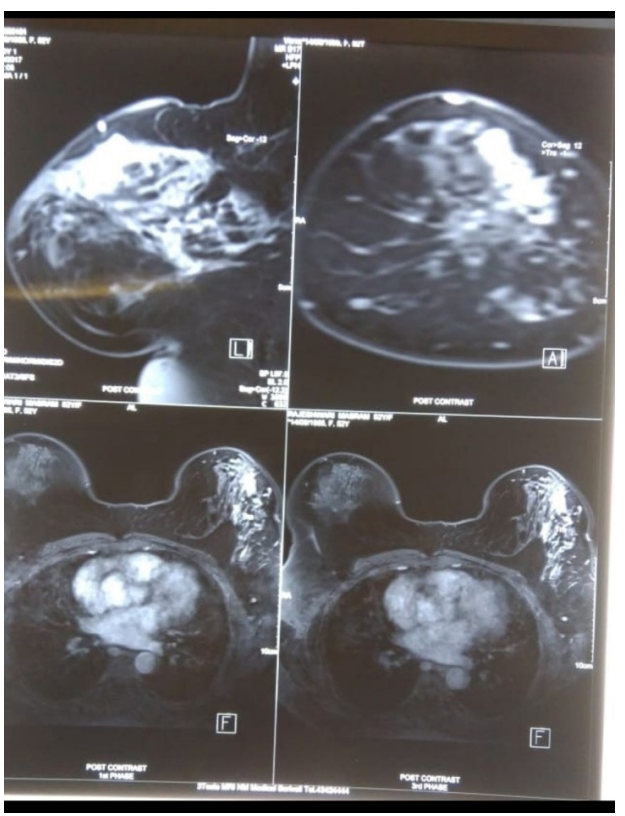

Figure 2 MRI showing micro calcification. 


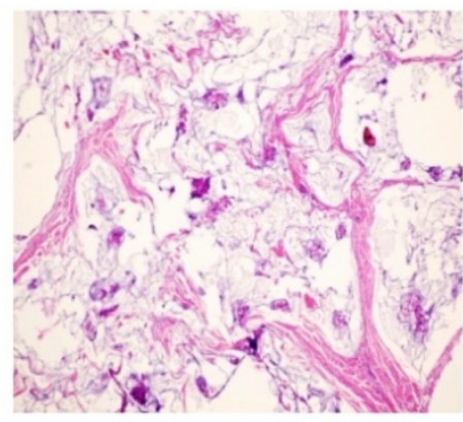

(a).

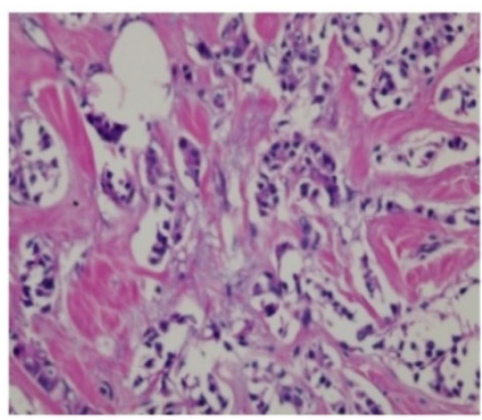

(b).

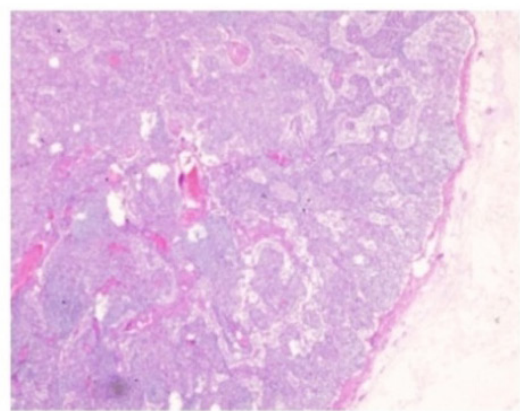

(c).

Figure 3 Histological appearance of (a) Mucinous carcinoma (b) Papillary carcinoma (c) Ductal carcinoma.

In our case study, three different histological types of breast carcinoma were found on left breast-mucinous carcinoma, invasive ductal carcinoma and intraductal papillary carcinoma. Mucinous carcinoma (MC) accounts for $4 \%$ of all invasive breast carcinomas ${ }^{2}$ and is commonly seen in perimenopausal and postmenopausal women. It is characterized by extracellular mucin. Although it is considered as an invasive tumor, $\mathrm{Rosai}^{3}$ has proposed that it is a form of in situ carcinoma whose production of mucin occurs towards the stroma, rather than towards the luminous surface. In this way mucin invades the stroma and separates the cells from its basal membrane, with two histological types: pure and mixed mucinous carcinoma, with different implications in diagnosis. It commonly presents with well-defined round shaped lesion on mammography and on ultrasound examination, it appears as isoechoic with the surrounding subcutaneous fat. These characteristics make it difficult to differentiate mucinous carcinoma from fibroadenoma. Pure mucinous carcinoma has good prognosis compared to the invasive variant.

Papillary breast cancer accounts for $0.5 \%$ of these invasive breast cancers, which often present with bloody nipple discharge or an abnormal mass on Ultrasound/Mammogram. ${ }^{4}$ Histologically there is proliferations of cells arranged around fibrovascular cores, grossly forming a circumscribed mass. ${ }^{5}$ On ultrasound, there is an intracystic mass or solid intraductal mass filling the duct with or without duct dilatation.

Invasive ductal carcinoma (IDC) is the most common histological variant of breast malignancy. ${ }^{6}$ Modified Bloom-Richardson grading system has 3 components: tubule formation, nuclear pleomorphism and mitotic count. Mammography is baseline investigation in breast disease screening owing to its simplicity, low cost and wide application and clarity in display of breast lumps' shape and boundary, and diagnosing sand-like calcification in lesions with high accuracy. ${ }^{7}$ In our case our clinical and radiological findings were discordant and we opted for a higher investigation. However in our case, MRI was done for more detailed analysis of cause of nipple discharge as mammogram findings were inconclusive. Breast MRI is mainly used among young women with high risk of malignancy, to know dimensions of the cancer, look for other tumors in the same or contralateral breast. ${ }^{8}$ In the present study, MRI helped us pick up a malignancy which was missed on mammogram and on further surgery we realised presence of 3 different variants of cancer in the same patient which is a unique factor.

\section{Conclusion}

Unilateral serosanguinous nipple discharge is a cause of concern always and needs full evaluation to rule out malignancy before we put these cases on active surveillance. Dynamic contrast-enhanced MRI (DCE MRI), which uses injectable gadolinium, is the most powerful method for detecting breast cancers. As this entity is rare, we need to have a high suspicion and accordingly investigate. Most of the available data comes from small series and case reports making it difficult to recommend management and treatment options. 3 different variants of histopathology on the same tumour is a rarity. Triple assessment and active surveillance help in reducing the chances of missing malignancy and improving patient survival and decreasing morbidity and mortality.

\section{Acknowledgments}

None.

\section{Conflicts of interest}

The authors declare that they have no conflict of interests.

\section{References}

1. Lakhani SR, Ellis IO, Schnitt SJ, et al. WHO Classification of Tumours of the Breast. 4th ed. IARC: Lyon; 2012.

2. Stephan P. What you need to know about breast cancer symptoms. About. com: Breast cancer; 2007.

3. Rosai J. Ackerman's Surgical Pathology. 10th ed. USA: Mosby; 2011 1699-1701 p.

4. Acevedo C, Amaya C, López-Guerra J. Rare breast tumors: Review of the literature. Reports of Practical Oncology \& Radiotherapy. 2014;19(4):267-274.

5. Collins LC, Schnitt SJ. Papillary lesions of the breast: selected diagnostic and management issues. Histopathology. 2008;52(1):20-29.

6. Ornia Rodriguez M, López Secades MA, Domínguez Iglesias F. Tumor mucinoso de mama: diagnóstico radiológico. SERAM; 2014.

7. Hao YP, Zhou SY, Liu RB, et al. Clustered microcalcification on mammogram-its value in the diagnosis of breast cancer. J Clin Radiol. 2001;20(1):7-10.

8. Dorria Saleh Salem, Rasha Mohamed Kamal, Sahar Mahmoud Mansour, et al. Breast imaging in the young: the role of magnetic resonance imaging in breast cancer screening, diagnosis and follow-up. J Thorac Dis. 2013;5(Suppl 1):S9-S18. 